Revue d'histoire de l'Amérique française

\#Q REVUE D.HISTOIRE DE L'AMÉRIQUE FRANÇAISE

\title{
"Nos routes se couvrent de touristes à la recherche de nos reliques du passé "
}

Les débuts de la Commission des monuments historiques (1922-1928)

\section{Pierre-Olivier Ouellet}

Volume 61, numéro 2, automne 2007

URI : https://id.erudit.org/iderudit/018061ar

DOI : https://doi.org/10.7202/018061ar

Aller au sommaire du numéro

Éditeur(s)

Institut d'histoire de l'Amérique française

ISSN

0035-2357 (imprimé)

1492-1383 (numérique)

Découvrir la revue

Citer cette note

Ouellet, P.-O. (2007). « Nos routes se couvrent de touristes à la recherche de nos reliques du passé " : les débuts de la Commission des monuments historiques (1922-1928). Revue d'histoire de l'Amérique française, 61(2), 235-251.

https://doi.org/10.7202/018061ar d'utilisation que vous pouvez consulter en ligne. 


\section{Note de recherche}

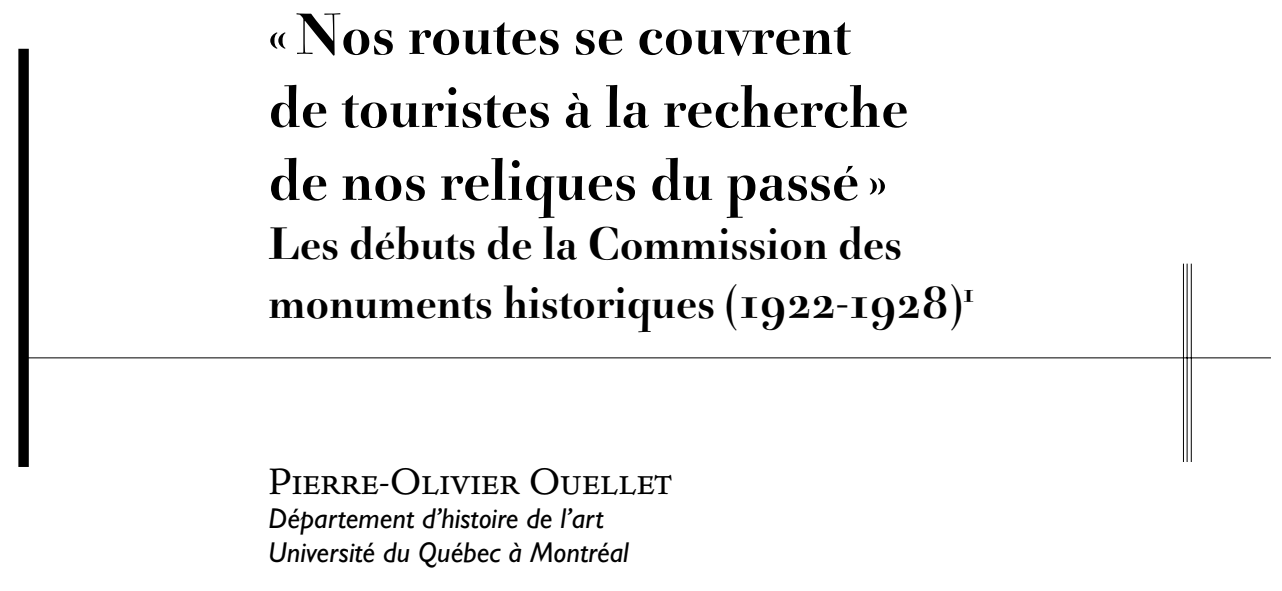

$\mathrm{E}^{\mathrm{n}}$ n 1922, le gouvernement, dirigé par le premier ministre libéral LouisAlexandre Taschereau (1867-1952), dépose la Loi des monuments historiques ou artistiques. Ce texte identifie, dans son préambule, les intentions originelles de l'État quant à la sauvegarde et à la mise en valeur des biens culturels :

Attendu que la conservation des monuments et des objets d'art historiques et artistiques est d'un intérêt national;

Attendu qu'il existe dans la province des monuments et des objets d'art dont le caractère historique ou artistique est incontestable ;

Attendu que le classement est la première condition de la conservation des monuments et des objets d'art ayant un intérêt historique ou artistique;

Attendu que le classement de ces monuments et de ces objets d'art s'impose $^{2}$.

1. Je remercie Mario Béland et Daniel Drouin, tous deux conservateurs de l'art ancien au Musée national des beaux-arts du Québec, pour leurs lectures rigoureuses des différentes versions de ce texte ainsi que Laurier Lacroix, professeur émérite à l'Université du Québec à Montréal, pour ses conseils au moment de l'élaboration de ma réflexion. Je suis également reconnaissant envers les évaluateurs anonymes de la Revue pour leurs suggestions et leurs commentaires très constructifs.

2. «Loi relative à la conservation des monuments et des objets d'art ayant un intérêt historique ou artistique ", Arrêtés en conseil ayant force de loi dans la province de Québec (Québec, Ls-A. Proulx, 1922), 149. 
La Loi étant adoptée et sanctionnée en mars 1922, le gouvernement met en place la Commission des monuments historiques composée du sous-secrétaire de la province nommé d'office, Charles-Joseph Simard (1877-1931), auquel s'ajoute Adélard Turgeon (1863-1930), conseiller législatif et premier président de l'organisme ${ }^{3}$. Quatre autres membres sont nommés, se distinguant par leur appartenance à des associations déjà préoccupées de conservation: Victor Morin (1865-1960), notaire, président de la Société Saint-Jean-Baptiste de Montréal et de la Société historique de Montréal; Édouard-Zotique Massicotte (1867-1947), avocat, archiviste aux Archives judiciaires de Montréal et président de la Société de Folklore; William Douw Lighthall (1857-1954), avocat et président de la Société d'archéologie et de numismatique de Montréal; ainsi que Pierre-Georges Roy (1870-1953), directeur-fondateur du Bulletin des recherches historiques et premier archiviste de la province.

Au cours des six années qui suivent, la Commission entreprend la fonte et l'installation de plaques commémoratives installées dans les espaces publics, publie quatre importants ouvrages illustrés, commande des sculptures de héros nationaux, etc., toutes des réalisations qui seront notamment saluées pour leurs valeurs patriotique et éducative ${ }^{4}$. C'est ce qu'en retiendra l'historiographie, alors que l'on présentera généralement l'organisme comme le gardien des traditions canadiennes-françaises à une époque marquée par les idéologies de conservation et de résistance à l'industrialisation et à la menace culturelle américaine ${ }^{5}$ (figure 1). Au cours

3. Turgeon était également le principal promoteur de la Loi de 1922. Alain Gelly, Louise Brunelle-Lavoie et Corneliu Kirjan, La passion du patrimoine: La Commission des biens culturels du Québec 1922-1994 (Sillery, Septentrion, 1995), 20; Robert Rumilly, Histoire de la province de Québec (Montréal, Éditions Chantecler, 1953), 26: 54.

4. Ferdinand Bélanger, "Dévoilement des statues de Boucher et de la Vérendrye», L'Action catholique, 29 septembre 1922, 8-10. L'auteur précise que par la tenue de cette cérémonie «la Commission des monuments historiques avait voulu faire une fête d'éducation patriotique» (p. 10). Sur ce même événement, voir les extraits des articles de La Presse et du Soleil reproduits dans: Commission des monuments historiques de la province de Québec, Premier rapport de la Commission des monuments historiques de la province de Québec 1922-1923 (Québec, Ls-A. Proulx, 1923), xii-xiv. En 1923, Louis-Athanase David (1882-1953) assure que la "commission établira sans conteste l'utilité de sa création en même temps que le travail patriotique qu'il est à accomplir ", dans Rapport du Secrétaire et registraire de la province de Québec 1922-1923 (Québec, La-A. Proulx, 1923), 9c.

5. Paul-Louis Martin, «Évolution de la vision collective du patrimoine au Québec, 1922-1985. Réflexions pour l'histoire culturelle contemporaine», dans Jacques Mathieu, dir., Étude de la construction de la mémoire collective des Québécois au XXe siècle: approches multidisciplinaires (Québec, CÉLAT, 1986), 144-150; Christian Vachon, «Des instruments de sauvegarde du patrimoine. Les recueils de Pierre-Georges Roy», Cap-aux-Diamants, 3,2 (été 1987): 45-47. Paul-Louis Martin, «La conservation du patrimoine culturel: origines et évolution", dans Les chemins de la mémoire 
Figure I

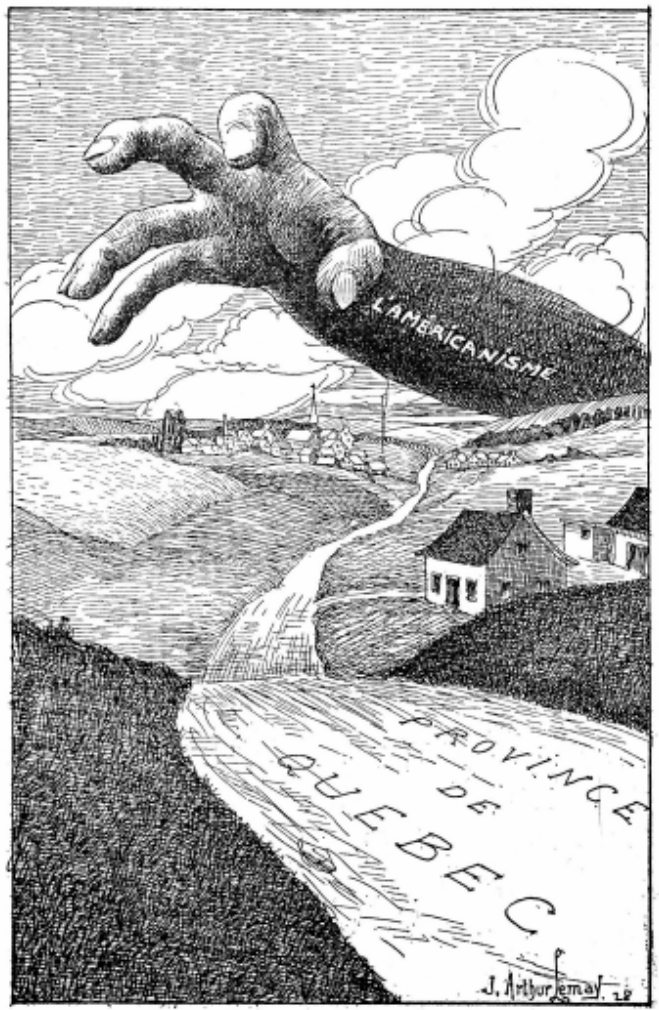

«La menace», illustration de J. Arthur Lemay (1900-1944) provenant de l'Almanach de la langue française, 14,4 (1928): 62 .

des dernières années, soulignons toutefois que ce rôle traditionnel a été nuancé. En effet, dès 1997, l'historien Charles Deblois-Martin rappelait brièvement que la multiplication d'inscriptions historiques aux abords des grandes routes du Québec s'interprétait comme un geste précurseur de la mise en relation entre le patrimoine et le tourisme ${ }^{6}$. Depuis, on reconnait,

(Québec, Les publications du Québec, 1990), 1: 6-10; A. Gelly, L. Brunelle-Lavoie et C. Kirjan, op. cit., 37-39. Ces derniers, comme ils le mentionnent en introduction, procèdent à l'examen de «la vision des législateurs sur la conservation [afin de] refléter le discours officiel de l’État» (p. 15), mais ne s'intéressent pas aux discontinuités de ce discours, dues au rôle touristique de la Commission.

6. Charles de Blois Martin, L'évolution des rapports entre les politiques du patrimoine et du tourisme au Québec, mémoire de maîtrise (science politique), Université Laval, 1997, 42; Charles de Blois Martin, "Survol historique des pratiques touristiques au Québec», Continuité, 81 (été 1999): 31. 
sans plus de détails, la participation de la Commission au développement touristique de la province ${ }^{7}$. Cela dit, la nature de cette implication demeure encore marquée par la méconnaissance des desseins des commissaires et du Secrétariat de la province qui chapeaute l'organisme. De fait, la Loi de 1922, constituant un discours d'intention, souligne seulement un enjeu de préservation. De même, les ouvrages de la Commission, réalisés entre 1923 et 1928, présentent plutôt un discours antimoderniste qui, a priori, s'accorde mal avec un désir d'attirer en grand nombre des touristes américains, lesquels sont souvent perçus comme des hérauts de la vie industrielle, de l'american way of life, doublés de pilleurs d'antiquités ${ }^{8}$. Pourtant, l'étude des archives, de même que la lecture des discours officiels de l'organisme et du secrétaire de la province Louis-Athanase David (1882-1953), permettent de mieux cerner l'importante place accordée au tourisme. Tout en mettant en lumière cet aspect du discours longtemps évité par l'historiographie afin de privilégier les nobles objectifs anticipés de conservation -, nous entreprendrons une lecture touristique de leurs travaux, en les situant et en les interprétant dans un contexte marqué par l'émergence d'un tourisme itinérant de masse.

\section{SUR LES TRAJECTOIRES DE MOBILITÉ DES TOURISTES: LES PLAQUES COMMÉMORATIVES}

Réalisation la plus manifeste d'une relation au tourisme, la création de plaques commémoratives fait partie des premiers projets de la Commission. Ainsi, dès sa seconde assemblée tenue le 6 octobre 1922, elle étudie «l'idée de placer des plaques commémoratives sur les sites historiques qui se rencontrent le long de nos routes de campagne si fréquentées aujourd'hui par les automobiles ${ }^{9} »$. Le premier rapport de l'organisme précise même que «ces inscriptions, rédigées dans les deux langues, signaleraient aux étrangers les sites d'un intérêt historique ${ }^{10}$ ». En 1923, le sous-secrétaire Simard se charge d'ailleurs de faire confectionner un modèle, lequel sera réalisé par le sculpteur français Jan Bailleul (actif au Canada entre 1914 et

7. Serge Gagnon, L'échiquier touristique québécois (Sainte-Foy, Presses de l'Université du Québec, 2003), 254.

8. Olivier Maurault, "Respect aux vieilles choses», La Revue nationale, 6,6 (juin 1924): 191 ; Hermas Bastien, "Gardons nos vieilles choses », La Revue nationale, 8,8 (août 1926): 229-231.

9. Québec, Bibliothèque et Archives nationales du Québec (BAnQ), Fonds Commission des biens culturels du Québec (E52), Commission des monuments historiques - procès-verbaux des réunions, 1993-12-005 / 31, "Procès-verbal, 6 octobre 1922 ».

10. Commission des monuments historiques de la province de Québec, Premier rapport de la Commission..., op. cit., $\mathrm{xv}$. 
1929), directeur de l'École des beaux-arts de Québec (figure 2). Ces plaques, installées le long des routes dès l'été de $1925^{11}$, produisent des résultats fort intéressants comme le précisent les membres de la Commission dans leur troisième rapport daté de 1926:

Disons ici que ces plaques sont très appréciées des touristes. La province de Québec est une terre historique par excellence. La plupart de nos anciennes paroisses ont vu des événements importants se dérouler sur leur territoire. Chacune aura son tour. Les inscriptions placées par notre Commission sur leurs principales voies de communications diront aux étrangers ce qu'elles ont été.

[Les plaques] sont des points de repère très appréciés des touristes. Elles éveillent leur curiosité et leur attention ${ }^{12}$.

Comme on le constate, la portée touristique de cette initiative n'est pas dissimulée, mais incluse dans le discours officiel de la Commission. Même dans une publication aussi populaire que l'Almanach du peuple publié chez Beauchemin, on proclame sans ambages que ces plaques ont été érigées "pour l'enseignement des touristes ${ }^{13}$ ». Étant donné les résultats positifs de cette entreprise, la Commission produit ainsi plus de 150 plaques commémoratives entre 1925 et $1928^{14}$. Comme les documents l'indiquent, celles-ci constituent alors des "points de repère " pour les touristes, c'està-dire des marqueurs pointant des attraits accessibles et communiquant de l'information sur le site ${ }^{15}$. De fait, placées en grande majorité aux abords des grands centres (Québec et Montréal), puis se diffusant ensuite le long de la vallée laurentienne selon les grandes routes, la disposition des plaques tient compte de la nouvelle réalité touristique de cette époque fordiste. Dans les années 1920, l'automobile et le développement de la voirie au Québec favorisent une expansion rapide d'un nouveau mouvement touristique: le tourisme itinérant. Ainsi, de plus en plus d'Améri-

11. Commission des monuments historiques de la province de Québec, Deuxième rapport de la Commission des monuments historiques de la province de Québec 1923-1925 (Québec, Ls-A. Proulx, 1925), xii, xiv, xviii-xxiv. En 1924, une inscription est apposée sur la maison natale d'Antoine GérinLajoie. Il semble néanmoins que ce soit à partir de l'été 1925 que les 50 premières plaques ont été installées à des points stratégiques le long des grandes routes du Québec.

12. Commission des monuments historiques de la province de Québec, Troisième rapport de la Commission des monuments historiques de la province de Québec 1925-1926 (Québec, Ls-A. Proulx, 1926), 8 et 12 .

13. "Commission des monuments historiques de la province de Québec», Almanach du peuple (Montréal, Librairie Beauchemin Limitée, 1927), 235.

14. Louis-Athanase David, Rapport du Secrétaire et registraire de la province de Québec 1927-28 (Québec, Ls-A. Proulx, 1928), 5u.

15. S. Gagnon, L'échiquier..., op. cit., 43. 


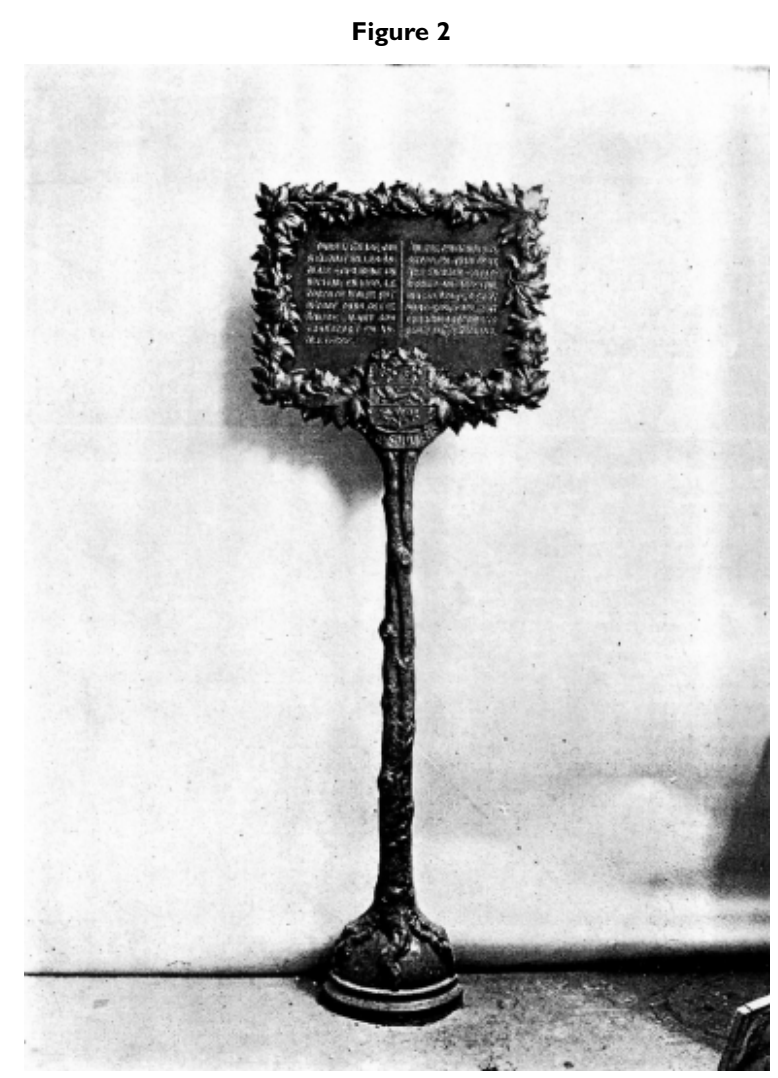

Légende: «Modèle des inscriptions historiques placées le long des routes de la province par la Commission des monuments historiques ", photographie provenant du Deuxième rapport de la Commission des monuments historiques de la province de Québec 1923-1925 (Québec, Ls-A. Proulx, 1925), xv.

cains traversent la frontière à bord de leurs voitures sans chevaux (figure 3), profitant d'une plus grande liberté dans leurs déplacements et dans la planification de leur séjour. Par exemple, en 1928, on recense environ 500000 voitures provenant des États-Unis et qui ont emprunté les routes de la province, soit quinze fois plus qu'en 1920 (tableau 1). Pour ces touristes autonomes dans leurs mouvements transitaires, les plaques ajoutent alors un aspect récréatif et culturel aux déplacements, venant rythmer leur parcours de diverses possibilités d'arrêts à des points d'intérêts historiques.

En 1927, le ministère de la Voirie va d'ailleurs favoriser la reconnaissance des plaques par les touristes lors de la réédition de l'ouvrage Québec, The French-Canadian Province. Dans ce guide unilingue anglophone tiré à 
Figure 3

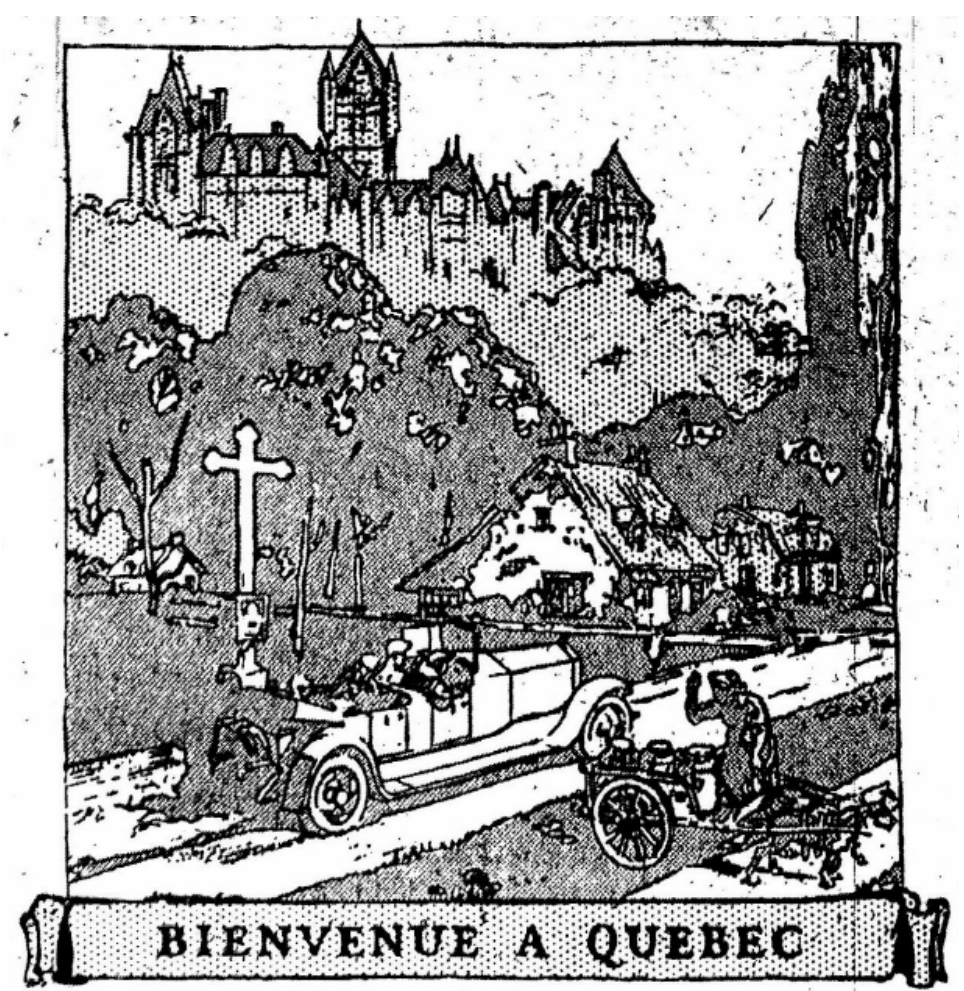

Légende: «Bienvenue à Québec», détail d'une publicité du Pacifique Canadien publiée dans le New York Times, 25 mai 1924, section 8, 14. Cette image est emblématique du contraste entre la modernité des touristes, circulant en automobile, et la tradition représentée par tous les éléments pittoresques du décor québécois, tels que la croix de chemin, les maisons rustiques et l'habitant. Bien en évidence, l'attelage de chiens tirant une charrette de bidons de lait fait même partie des curiosités du Québec. Ce motif est d'ailleurs utilisé et répété, dès la fin des années 1820, dans de nombreuses aquarelles de l'artiste militaire James Pattison Cockburn (1779-1847) destinées aux visiteurs de la capitale.

50000 exemplaires ${ }^{16}$ et distribué gratuitement à la frontière canadoaméricaine, on y remarque l'illustration d'une plaque commémorative réalisée par la Commission de même qu'une liste des textes gravés sur ces plaques $^{17}$ (figure 4). Destiné directement aux touristes, ce guide révèle ainsi l'existence des plaques et facilite leur repérage. Il confirme aussi les

16. Ministère de la Voirie, Rapport du Ministère de la voirie: rapport de 1927 (Québec, Ls-A. Proulx, 1927), 50.

17. Ministère de la Voirie, Québec, The French-Canadian Province (Québec, Roads Department, mars 1927), 10-15. 
Tableau I

Nombre d'automobiles de tourisme ayant traversé

la frontière entre 1920 et 1928

$\begin{array}{cc}\text { Année } & \text { Automobiles } \\ 1920 & 31918 \\ 1921 & 41957 \\ 1922 & 95163 \\ 1923 & 130099 \\ 1924 & 199008 \\ 1925 & 259959 \\ 1926 & 265848 \\ 1927 & 417561 \\ 1928 & 500000\end{array}$

Source: Ministère de la Voirie, Sur les routes du Québec : guide du touriste (Québec, Ministère de la Voirie et des Mines, novembre 1929), 49.

rôles matériel (comme instrument indicatif) et informatif (encourageant la création d'itinéraires de voyage) assumés par ces signes. En somme, par la rédaction des inscriptions et la mise en place des plaques à proximité des routes, la Commission rend visible des sites et des attraits culturels accessibles, particulièrement appropriés pour un tourisme itinérant en pleine émergence. Plus encore, ces plaques commémoratives constituent une véritable amorce d'organisation culturelle du territoire en fonction des trajectoires de mobilité empruntées par les touristes.

\section{INVENTAIRES ILLUSTRÉS: OUTILS DE CONNAISSANCE, DE PROMOTION ET DE VALORISATION DES ATTRAITS TOURISTIQUES}

Entre 1923 et 1927, le secrétaire de la Commission, Pierre-Georges Roy, signe les trois inventaires illustrés de l'organisme, soit Les monuments commémoratifs de la province de Québec ${ }^{18}$, Les vieilles églises de la province de Québec: $1647-1800^{19}$, puis Vieux manoirs, vieilles maisons ${ }^{20}$. L'abondant corpus

18. Pierre-Georges Roy, Les monuments commémoratifs de la province de Québec (Québec, Commission des monuments historiques de la province de Québec, 1923), 2 tomes. L'auteur avait commencé cet ouvrage avant 1918. Voir Bernard Weilbrenner, «Pierre-Georges Roy et le Bureau des archives de la Province 1920-1925», Archives, 21,1 (1989): 22.

19. Pierre-Georges Roy, Les vieilles églises de la province de Québec: 1647-1800 (Québec, Commission des monuments historiques de la province de Québec, 1925), 323 p. Cet ouvrage, comme les Vieux manoirs, vieilles maisons, est probablement le fruit du travail de tous les membres de la Commission des monuments historiques. Toutefois, Pierre-Georges Roy est généralement désigné comme le seul rédacteur de ce travail.

20. Pierre-Georges Roy, Vieux manoirs, vieilles maisons (Québec, Commission des monuments historiques de la province de Québec, 1927), 376 p. 


\title{
Figure 4
}

\section{HISTORIC INSCRIPTIONS}

\author{
PLACED ALONG THE HIGHWAYS OF THE \\ PROVINCE OF QUÉBEC
}

These inscriptions are bilingual. Only the English version is given here.

Benumont (Bellechasse). - In June, 1759, Monckton used this parish church for affixing the notice which history records.

Beaumont (Bellechasse). - Lord Elgin, Governor of Canada from 1847 to 1854 , rested from the care of office here.

Beauport (2uébec). - Montcalm had his headquarters close by, in the Duchesnay seigniorial manor, during the summer of 1759 .

Beauport (2uébec). - The hero of Chateauguay, Charles-Michel de Salaberry, was born here, November 18,1778 .

BerthIBR EN BAs seigniory of Berthier, Berthier, of the Carignan 1672 , passed to the Family.

Berthier en Haut iory of Berthier was Randin, 29 October, thier, an officer in the Regi from Randin.

Berthier en Haut (Bet in 1876 by the seignior, Cuth Protestant worship in the Pro

Boucherville (Chambly), Fontaine was born here, 4 June 1864 .

CaP SAINTIG IG A E the seigniorial mill of the lotte,built in the middle of
(Montmagny). - The granted to the Sieur Regiment, 29 October, Bergères de Rigauville

(Berthier), - The seign granted to the Sieur 1672. Alexander Ber. ment of Carignan, bought it

thier). - This chapel was built bert. It was the first place of vince of Québec.

- Sir Louis Hippolyte La October, 1807. He died, 26

(Montmagny). - Site of fief and seigniory of Vince. the seventeenth century.

Model of Historic Inscription.

PAGE TEN

Légende: "Historic inscriptions placed along the highways of the province of Québec», page tirée du guide Québec, The French-Canadian Province (Québec, Roads Department, mars 1927), 10.

documentaire - auquel est ajouté un impressionnant corpus visuel constitué notamment de photographies réalisées par Edgar Gariépy (18811956) - est offert au public sous la forme d'ouvrages très abondamment illustrés pour l'époque et se distinguant par la qualité de l'édition.

Avec ces ouvrages qui recensent les biens et qui compilent les informations historiques sur ceux-ci, les commissaires produisent des instruments de premier ordre afin d'alimenter le travail des promoteurs touristiques. Par exemple, l'École des guides historiques de Québec, à peine ouverte 
en 1924, offre trois cours sur les monuments commémoratifs dispensés par Alonzo Cinq-Mars (1881-1969) ${ }^{21}$. Le premier inventaire illustré de la Commission présente ainsi une synthèse exceptionnelle sur le sujet, assurant une connaissance et une reconnaissance appropriées des monuments à une époque où l'on fustige le travail des guides traditionnels de la Vieille Capitale (les cochers), incapables, semble-t-il, d'identifier correctement les différents personnages historiques statufiés ${ }^{22}$. Notons aussi la parution du guide Voyez Québec d'abord: See Québec First!, publié en 1926 par le ministère de la Voirie. Sa bibliographie comporte cinq livres dont les deux ouvrages de la Commission parus à ce moment, soit Les monuments commémoratifs de la province de Québec et Les vieilles églises de la province de Québec: 1647-180023. Les informations compilées par les commissaires semblent ainsi devenir une source essentielle au développement des connaissances liées au tourisme.

D’ailleurs, les membres de la Commission démontrent une nette compréhension de ce rôle dans leur troisième rapport:

[les] volumes publiés par la Commission des Monuments Historiques ont contribué - on a bien voulu nous le dire de toutes parts - à faire connaitre notre province à l'étranger et à attirer dans nos villes et nos campagnes des milliers de touristes américains désireux de voir nos trésors historiques de toutes sortes ${ }^{24}$.

Ainsi, les inventaires de l'organisme participent directement à la reconnaissance des biens historiques de la province. Cette promotion à l'extérieur du Québec est de plus facilitée par la traduction anglaise des ouvrages Les vieilles églises de la province de Québec: 1647-1800 et de Vieux manoirs, vieilles maisons. Ainsi, le Times de Londres publie en 1927 une

21. «Les guides historiques", Le Terroir, 4,9 (janvier 1924): 373. De manière très synchronisée, dans le même numéro du Terroir, une revue de littérature traite du premier inventaire illustré de la Commission: "Quant au rapport du Secrétaire de la Commission des Monuments Historiques, il se compose de deux forts volumes abondamment illustrés contenant l'historique et la photographie de chacun des 174 monuments commémoratifs que nous comptons dans la province de Québec, trente-sept dans Québec, quarante-huit dans Montréal et le reste dans les autres parties de la province» (p. 376).

22. À ce sujet, lire l'article de Jean-Charles Harvey, "Nos guides historiques à Québec», Le Terroir, 4,6 (octobre 1923): 233-235.

23. Ministère de la Voirie, Voyez Québec d'abord: See Québec First! (Québec, Ministère de la Voirie, mai 1926), 8.

24. Commission des monuments historiques de la province de Québec, Troisième rapport de la Commission..., op. cit., 12. Ces volumes sont alors: les deux tomes de Les monuments commémoratifs de la province de Québec; Les vieilles églises de la province de Québec: 1647-1800; et la traduction anglaise de ce dernier livre. 
appréciation de The Old Churches of The Province of Quebec: 1647-180025. La même année, l'éditeur Louis Carrier (1898-1961) organise une exposition de livres canadiens-français lors de la convention de l'American Library Association tenue à Toronto ${ }^{26}$. Réalisée sous le patronage de Louis-Athanase David et avec la collaboration, notamment, de trois membres de la Commission, l'exposition présente entre autres les ouvrages de l'organisme parmi les rares livres traduits et donc accessibles aux anglophones unilingues.

Mais le ton de ces documents, en particulier des introductions, pouvaitil vraiment favoriser le tourisme? Par exemple, dans Les vieilles églises de la province de Québec: 1647-1800, les commissaires regrettent la disparition des monuments disparus et accusent «la manie de détruire et l'amour de la nouveauté27 $»$. Dans les Vieux manoirs, vieilles maisons, on oppose la vieille habitation, symbole de l'âme de tout un peuple, aux maisons modernes, perçues comme étrangères. Comme le questionnent les commissaires: "sommes-nous chez nous dans nos maisons modernes comme nos ancêtres étaient chez eux dans leurs vieux logis aux divisions si simples et si familiales ${ }^{28}$ ?» Les commissaires investissent ces architectures de dimensions nationaliste, nostalgique et idéalisante du passé dans un discours antimoderniste. Dans une attitude de repli sur soi face aux rapides transformations de l'époque industrielle, les biens historiques deviennent ainsi des reliques identitaires à chérir et à préserver. Mais cette écriture que l'on croit d'abord rebutante pour le voyageur provenant des cités modernes et parcourant l'Amérique au moyen de son automobile constitue en fait le meilleur moyen de valoriser les attraits de la province. En effet, rappelons qu'en Allemagne, à partir de 1913, le guide touristique Baedeker dirige son attention, et celle de ses lecteurs, sur les sites et les biens représentatifs de la nation. Les monuments et les bâtiments historiques constituent alors les premiers éléments d'intérêt dans la recherche et la consommation d'une image nationale jugée authentique ${ }^{29}$. L'antimodernisme et les racines de l'identité nationale, matérialisées par

25. L'article du Times est reproduit dans: Commission des monuments historiques de la province de Québec, Troisième rapport de la Commission..., op. cit., 22-23.

26. Louis Carrier, The Books of French Canada, an Exhibit Prepared for The Annual Meeting of the American Library Association, Toronto, June 1927 (Montréal, Louis Carrier et les Éditions du Mercure, 1927), 47 p.

27. P.-G. Roy, Les vieilles églises..., op. cit., vi.

28. P.-G. Roy, Vieux manoirs..., op. cit., vii.

29. Rudy Koshar, "What Ought to Be Seen” : Tourists' Guidebooks and National Identities in Modern Germany and Europe ", Journal of Contemporary History, 33,3 (July 1998): 332-339. 
certains biens, permettent ainsi au touriste d'espérer une expérience élevée au-dessus du quotidien et des seuls intérêts modernes, industriels ou économiques du territoire visité. Dans ce même esprit, en se portant garant des qualités historiques et en construisant le caractère identitaire des monuments, les commissaires, spécialistes de la culture nationale, jouent donc un rôle productif dans la valorisation des attraits de la province.

\section{L'ÎLE D'ORLÉANS}

Dernier ouvrage produit par la Commission dans les années 1920, L'île d'Orléans ${ }^{30}$ est publié en 1928. Contrairement aux ouvrages précédents qui prennent la forme d'inventaires, ce livre se présente plutôt comme une monographie composée d'une série d'histoires et d'anecdotes folkloriques. Ainsi, aux monuments matériels et aux biens culturels à classer, on préfère raconter l'histoire d'une magnifique île du Saint-Laurent, laquelle sera de plus comparée à un Éden, un lieu de survivance des traditions n'ayant pas changé depuis trois cents ans ${ }^{31}$. Pourtant, comme l'affirmait Massicotte dans une lettre adressée à Roy en 1927, les objets vulnérables et à inventorier par la Commission étaient encore bien nombreux:

Cher monsieur Roy,

Relativement aux prochains rapports de la C.d.m.h., voici ce que je pense.

Avant de se borner à de simples monographies de localités, ne vaut-il pas mieux épuiser les sujets d'ensemble et qui intéressent les gens d'un bout à l'autre du Canada français?

Considérez par exemples les sujets suivants:

Les églises disparues et ce qui en reste [...].

Nos croix de chemin [...].

Nos monuments intérieurs [...].

Portraits \& tableaux historiques [...].

D’ailleurs, il y a dans les sujets que je signale des trésors pour l'histoire générale et qui peuvent disparaître d'un jour à l'autre ${ }^{32}$.

À la lumière de ce rappel et de cet avertissement, il semble alors difficile de justifier l'ouvrage sur l'île d'Orléans par l'organisme dans une perspec-

30. Pierre-Georges Roy, L'̂̂le d'Orléans (Québec, Ls.-A. Proulx, 1928), 505 p.

31. Ibid., v-vi.

32. Québec, Bibliothèque et Archives nationales du Québec (BAnQ), Fonds Archives nationales du Québec (E53), Correspondance, 1919-00-06 / 24, art. 26, «Lettre de EZ Massicotte à PG Roy, 22 janvier 1927 ». 
tive univoque de conservation des monuments. En réalité, ce livre constitue plutôt un cas exemplaire des efforts touristiques de la Commission, reléguant leur mission initiale en marge de leurs préoccupations. En effet, dès 1925, Pierre-Georges Roy est bien averti du potentiel touristique de l'île d'Orléans. Il prodigue alors quelques conseils éclairés à un certain monsieur Juneau lui ayant soumis un parcours de l'île:

J'ai lu avec le plus vif intérêt l'itinéraire du tour de l'île que vous m’avez communiqué [...]. Voulez-vous me permettre une suggestion? Pourquoi ne publieriez-vous pas cet itinéraire en anglais et en français sur la même feuille côte à côte? [...] D'ailleurs, les Américains viennent dans notre Province pour voir ce qu'ils n'ont pas chez eux, c'est-à-dire du français, des choses françaises et des villages français. Un itinéraire bilingue leur sera agréable, soyez-en certain $^{33}$.

Deux ans plus tard, les tours de l'île d'Orléans font même partie des premiers circuits touristiques de la province réalisés «sous le signe de l'identité ${ }^{34} »$. De fait, comme l'invite à croire un article de Jules Ledoux publié en 1924 dans la Revue nationale, il semble que les Canadiens français aient été bien avertis et conscientisés quant à la valeur touristique de leurs traditions et de leur langue: "Plus nous serons français et catholiques, plus nous offrirons quelque chose de nouveau, d'exotique, de rare aux Américains. [...] il est pratique de garder les coutumes de nos aïeux, notre langue, notre foi, notre âme française. C'est une leçon et un argument $t^{35}$.» Ainsi, en rédigeant un ouvrage sur l'île d'Orléans qui mise sur la promotion du fait français et la création d'une image mythique d'un territoire préindustriel où survivent les traditions, les commissaires ne peuvent

33. Québec, Bibliothèque et Archives nationales du Québec (BAnQ), Fonds Archives nationales du Québec (E53), Correspondance, 1919-00-006 / 24, art. 25, "Lettre de PG Roy à M. Juneau, 5 juin $1925 »$.

34. Serge Gagnon, «L'émergence de l’identité rurale et l’intervention de l’État québécois en tourisme (1920-1940)", Téoros, 20, 3 (automne 2001): 25.

35. Jules Ledoux, "Le tourisme, ses avantages et les devoirs qu'il nous impose», La Revue nationale, 6,10 (octobre 1924): 333-334. L'Almanach du peuple publie, en 1925, un article d'un auteur anonyme qui présente une opinion similaire: "Pourquoi ne pas attribuer tout simplement ce débordement de visiteurs à l'attrait bien particulier de la province de Québec, attrait sans doute accru par d'excellentes routes et des paysages ne le cédant à ceux d'aucun autre pays, mais qui s'explique surtout, croyons-nous, par le fait que notre province est toujours restée, en dépit de l'énorme ambiance anglo-saxonne, fidèle à ses origines de Nouvelle-France, qui en ont fait une contrée bien à part dans toute l'uniformité de l'Amérique de langue anglaise. En fait, ne serait-ce pas ici une autre et nouvelle France, dans toute l'acceptation vraie du mot, qui exerce le charme qui lui est particulier à l'endroit de ses voisins anglo-saxome [sic]». Voir «La Nouvelle-France et le tourisme», Almanach du peuple (Montréal, Librairie Beauchemin limitée, 1925), 210. 
convier plus explicitement les touristes à visiter ce lieu folklorique, promettant un dépaysement. Plus encore, ils véhiculent alors un discours et une image de l'île tout à fait similaires à ceux développés par les promoteurs touristiques de l'île-du-Prince-Édouard au début des années 1920, misant sur le garden myth, la terre oubliée par le temps, et son folklore $^{36}$.

La facture du livre est également très révélatrice. On édite à grands frais un ouvrage attrayant ${ }^{37}$. Comme nous en informe la correspondance de Roy, c'est le premier ministre du Québec lui-même qui insiste sur cet aspect: «M. Taschereau nous a dit de le faire beau et nous ne négligeons rien $^{38}$.» Des artistes du Québec sont ainsi mis à contribution, produisant les nombreuses illustrations du volume. Parmi ceux-ci, soulignons le travail de l'artiste canadien Horatio Walker (1858-1938) - établi en permanence sur l'île vers 1919 - dont les œuvres parviennent parfaitement à enrichir l'enjeu touristique de l'ouvrage. En effet, en plus d'être associé au marché de l'art américain, et de jouir à ce moment de sa carrière d'une «solide renommée outre-frontière ${ }^{39}$ », le peintre de l'île effectuait depuis plusieurs années une artialisation de ce terroir ${ }^{40}$. Les scènes traditionnelles illustrées par Walker traduisent alors une époque qui semblait révolue (figures 5 et 6), et procèdent de plus à une saisie des valeurs affectives et esthétiques des lieux, transmettant le désir de voir le modèle original ${ }^{41}$.

Tout comme Les vieilles églises de la province de Québec: 1647-1800 et les Vieux manoirs, vieilles maisons, le livre L'île d'Orléans bénéficiera aussi d'une traduction anglaise afin de rejoindre plus facilement les Américains et les Canadiens anglais. La liste d'envoi des volumes traduits est, à cet égard, très informative ${ }^{42}$. En tout, 112 exemplaires du volume sont distribués,

36. Matthew McRae, "The Romance of Canada: Tourism and Nationalism Meet in Charlottetown, 1939", Acadiensis, 34,2 (printemps 2005): 27-28.

37. À ce sujet, Luc Noppen écrit que «Lîle d’Orléans de Pierre-Georges Roy se range dans la catégorie des "beaux livres" ». [...] De plus, avec ce livre, la commission innove dans le domaine de l'édition de luxe. «[...] Jusqu'alors l'édition n'avait offert que fort peu d'ouvrages d'une présentation si soignée : iconographie aussi abondante que variée, typographie nette, papier d'excellente qualité. » Voir "L'ouvrage», L'île d'Orléans (Québec, Librairie Garneau, 1976), 510-511.

38. Québec, Bibliothèque et Archives nationales du Québec (BAnQ), Fonds Famille Roy (P608), P608, S1, SS1, «Lettre de PG Roy à Antoine Roy, $1^{\text {er }}$ novembre 1927 ».

39. David Karel, Horatio Walker (Québec, Musée du Québec/Fides, 1986), 92.

40. Lucie K. Morisset, «Voyage au pays de l'identité. De la définition d'un paysage touristique à la création de la spécificité culturelle canadienne-française», L'espace touristique (Sainte-Foy, Presses de l’Université du Québec, 1999), 220-222.

41. S. Gagnon, L'échiquier..., op. cit., 50-52.

42. Québec, Bibliothèque et Archives nationales du Québec (BAnQ), Fonds Archives nationales du Québec (E53), E53, S7, SS7, «Liste pour l'envoi de volumes anglais The Isle of Orleans». 
Figure 5

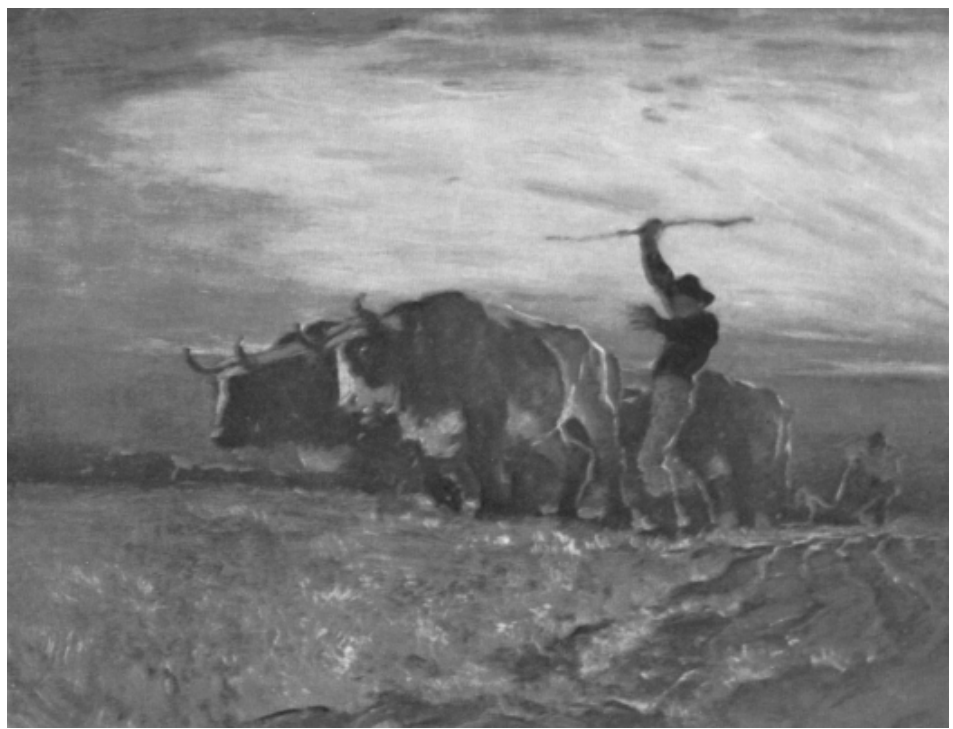

Légende : «Labourage à l'aube», illustration en couleurs de l’œuvre de Horatio Walker insérée entre les pages 96 et 97 du livre L'île d'Orléans (Québec, Ls.-A. Proulx, 1928).

Figure 6

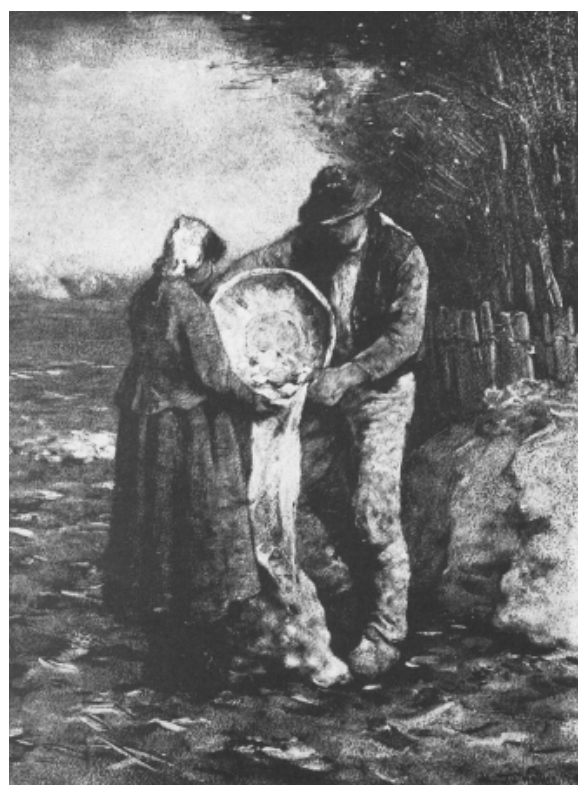

Légende : «La récolte des patates», illustration en noir et blanc de l'œuvre de Horatio Walker publiée dans le livre L'île d'Orléans (Québec, Ls.-A. Proulx, 1928), 392. 
dont 66 aux États-Unis, essentiellement aux États de la côte Est, dont New York et le New Jersey. Les chambres de commerce et les journaux américains, comme The New York Times, sont ciblés. Pour les 46 exemplaires envoyés au Canada anglais, 34 d'entre eux sont destinés à l'Ontario, dont 14 aux journaux de cette seule province. En donnant tous ces exemplaires aux «voisins » du Québec, et en particulier à la presse écrite, les commissaires organisent donc une évidente campagne de promotion de l'île, exportant à l'étranger une image de soi traditionnelle et d'essence française. Bref, la réalisation de ce volume, qui sera d'ailleurs l'ouvrage culminant de la Commission, laisse soupçonner qu'un large public auquel il s'adresse... se trouve à l'extérieur du Québec.

\section{CONCLUSION}

Dans un bilan dressé en 1928, Louis-Athanase David écrivait sans ambiguiité que les livres intitulés

«Les Monuments Historiques», «Les vieilles Églises», «Vieux Manoirs, Vieilles Maisons» et «L'île d'Orléans » [que la Commission] nous présente en dernier lieu, grâce à leur belle tenue historique et artistique, donnent, dès à présent, des résultats que nous n'attendions pas si rapides. C'est, répète-t-on à satiété, la propagande la plus efficace que puisse entreprendre un gouvernement. Il est déjà facile de s'en rendre compte. Nos routes se couvrent de touristes à la recherche de nos reliques du passé ${ }^{43}$.

Séparé d'à peine six ans de la Loi de 1922, ce rapport du secrétaire de la province confirme bien qu'en parallèle avec l'idéal de conservation de sa loi constitutive, l'organisme a développé un important mandat relatif au développement touristique. La Commission a donc participé à la construction des attraits culturels du Québec à une époque marquée par la mobilité et l'émergence d'un tourisme itinérant de masse. Cela étant, avançons également que cette préoccupation touristique n’était pas désintéressée. De fait, les commissaires étaient bien conscients que le tourisme permettait un accroissement considérable des revenus de l'État et enrichissait la province. À ce sujet, ils écrivaient dans leur deuxième rapport publié en 1925 : «Les milliers de citoyens des États-Unis qui, chaque année, traversent la frontière, laissent des sommes considérables dans nos villes et nos campagnes. Tout le monde profite de cette manne ${ }^{44}$.» Les répercussions

43. L.-A. David, Rapport du Secrétaire... 1927-28, op. cit., 5ss.

44. Commission des monuments historiques de la province de Québec, Deuxième rapport de la Commission..., op. cit., xvi-xvii. 
économiques dues au tourisme sont en effet remarquables. Ainsi, les Américains dépensent 15 millions de dollars dans la province en $1920^{45}$ et cette somme triple en 1925, atteignant 45 millions $^{46}$. Ce dernier montant représente alors le double de la valeur des produits de l'industrie minière se chiffrant, la même année, à près de 22500000 dollars ${ }^{47}$. Face à ces montants faramineux, on cerne davantage l'importance pour le gouvernement provincial d'entretenir cette industrie en plein essor, ce qui explique probablement en partie l'implication touristique de la Commission au cours de ses premières années d'existence. Cela dit, ce double discours, axé à la fois sur la conservation d'une culture matérielle investie de valeurs traditionnelles et sur son usage touristique, ne constitue pas pour autant une contradiction si manifeste. En effet, rappelons que selon l'historien Fernand Harvey, la "politique» culturelle de David consistait surtout à effectuer une conciliation entre «la tradition culturelle et la modernité économique ${ }^{48}$ ». La valorisation historique et la mise en tourisme des monuments ne pouvaient alors être plus représentatives de cette approche. Finalement, en regard de ces informations, demandons-nous si un autre grand projet du gouvernement Taschereau, soit la construction du Musée de la province sur le parc des Champs-de-Bataille, ne répondait pas également à ces mêmes préoccupations ${ }^{49}$ ?

45. Robert Prévost, Trois siècles de tourisme au Québec (Sillery, Septentrion, 2000), 81.

46. Ministère de la Voirie, Ministère de la voirie: rapport de 1926 (Québec, Ls-A. Proulx, 1926), 36.

47. "Le Canada», Almanach du peuple (Montréal, Librairie Beauchemin Limitée, 1927), 141.

48. Fernand Harvey, "La politique culturelle d'Athanase David, 1919-1936", Les cahiers des dix, 57 (2003): 79.

49. Notons également que les deux premiers directeurs du Musée de la province, ouvert en 1933, ont été le président (Simard) et le secrétaire (Roy) de la Commission. John R. Porter, «La collection du Musée national des beaux-arts du Québec », La collection du Musée national des beauxarts du Québec. Une histoire de l'art du Québec (Québec, Musée national des beaux-arts du Québec, 2004), 9-10. 